\title{
Pensionsplanung - was ist zu tun?
}

\author{
Mit zunehmendem Alter drängt sich immer mehr die Frage auf, wie das finan- \\ zielle Umfeld für die Zeit nach der Tätigkeit als praktizierender Arzt gestaltet \\ werden soll.
}

\section{Peter Heidelberger*}

Der Autor ist Versicherungsfachmann mit eidg. Fachausweis und Finanzplaner mit eidg. Fachausweis.
Korrespondenz:

Peter Heidelberger

Heidelberger Consulting AG

FMH Insurance Services

Marktgasse 61

CH-9500 Wil SG

Tel. 0719118135

Fax 0719118136

peter.heidelberger@fmhinsurance.ch

\section{Wie hoch wird mein Einkommen sein? Hält meine Vorsorge, was versprochen wurde? Kann ich den gewohnten Lebens- standard halten?}

Die Umstände, die zur Beantwortung dieser Fragen berücksichtigt werden müssen, können sehr unterschiedlich sein. So stellt sich dem Spitalarzt, der im Arbeitnehmerverhältnis angestellt ist, eher die Frage, wie seine Pensionskasse in bezug auf Auskauf der Frühpensionierung oder die Möglichkeit der Kapitaloption ausgestaltet ist, während beim selbständig praktizierenden Arzt die Regelung der Praxisnachfolge eine wichtige Rolle einnimmt, da der Verkaufserlös auch zur Altersvorsorge beiträgt. Ist ein Arzt selbständig und im Angestelltenverhältnis, ist eine sorgfältige Koordination der verschiedenen Vorsorgeeinrichtungen unerlässlich. Doch was gilt es alles zu beachten, und wie kann die eigene Situation optimiert werden? Eine gutstrukturierte und umfassende Pensionsplanung sollte folgende Themen beinhalten: Vorsorge, Anlagen, Immobilien, Steuern und Nachfolge. Die einzelnen Themen sind stark miteinander verknüpft und müssen immer im gesamten betrachtet werden.

Nachfolgend einige Informationen und Tips, was Sie in bezug auf Ihre Pensionierungsplanung beachten sollten.

\section{Vorsorge}

AHV-Beiträge werden auch noch auf Honorareinnahmen, die nach der Aufgabe der Praxis und nach dem 65. bzw. 64. Geburtstag anfallen, erhoben. Sollten Sie vor dem regulären AHV-Pensionsalter in Pension gehen, werden AHV-Beiträge aufgrund des Vermögens und des 20fachen Renteneinkommens erhoben. Sie bleiben AHV-beitragspflichtig bis zum 65. bzw. 64. Geburtstag.

Pensionskassenkapital, mit dem Kapitalbezug werden zwei Risiken selbst übernommen: das Anlagerisiko (Börse, Zinsrisiko usw.) und das Langlebigkeitsrisiko (wie lange muss das Kapital

\section{Tabelle 1}

Berechnung AHV-Beiträge (in Fr.)

Vermögen

900000

Renten aus Pensionskasse

1200000

$(60000 \times 20)$

massgebendes Vermögen

2100000

Jahresbeitrag pro Ehegatte

2020

( $1 / 2$ massgebendes Vermögen)

\section{Tabelle 2}

Kapital vs. Rente (in Fr.)

Pensionskassenkapital

900000

Rente aus Pensionskasse 6,8\%

61200

abzüglich jährliche Steuerbelastung

$-12700$

Netto Rente

48500

Kapitalauszahlung

900000

abzüglich einmalige Steuer

$-121000$

Netto Kapital

779000

Bei einem jährlichen Bezug von Fr. 48500 und einer Rendite von $3 \%$ ist das Kapital in 23 Jahren vollständig aufgebraucht (Steuerdaten Kanton Zürich).

reichen?). Stellen Sie sich die Frage, ob Sie diese Risiken tragen wollen und können.

Die von Vertrauenspartnern FMH Insurance Services angebotenen Pensionskassenlösungen beinhalten alle die Möglichkeit einer Kapitaloption, jedoch nicht alle Pensionskassen z.B. der Spitäler oder Kantone bieten diese Form der Altersleistung an.

\section{Anlagen}

Sind Vermögenswerte und Wertschriften vorhanden oder werden zukünftig durch den Verkauf der Praxis, den Kapitalbezug aus der Pensionskasse oder der 3. Säule freie Mittel zur Verfügung stehen, fällt der sorgfältigen Investition dieser Gelder eine wichtige Rolle zu. Stellen 
Abbildung 1

Aufbau und Entnahme der verschiedenen Anlagegefässe.

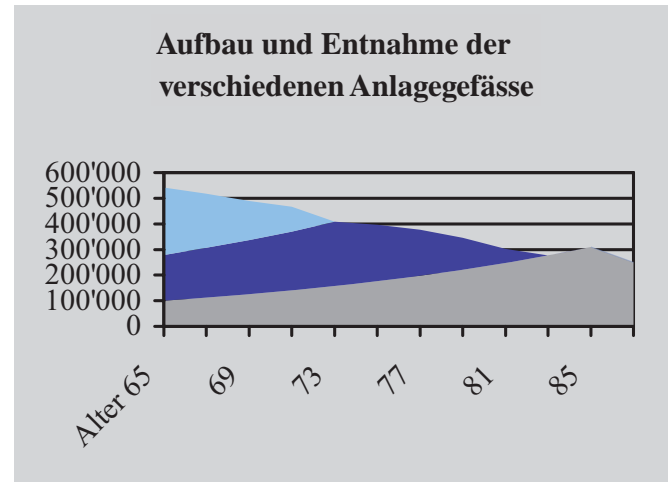

Abbildung 2

Kapitalsteuer.

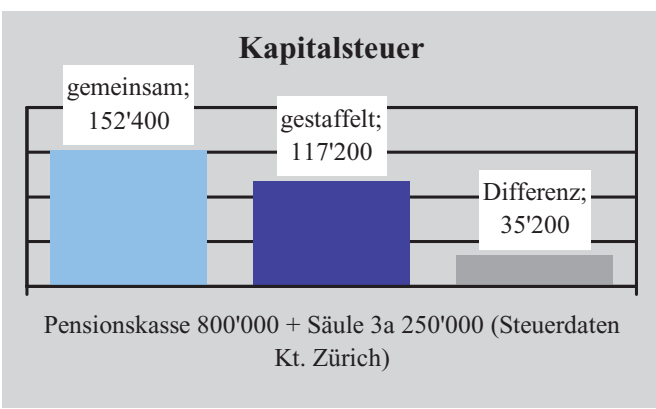

Sie sich die Frage, wieviel Einkommen Sie aus dem Vermögen zusätzlich zu den Renteneinkommen benötigen. Sie müssen nicht das ganze Vermögen jederzeit liquid halten, bilden Sie verschiedene Anlagegefässe mit verschiedenen Anlagehorizonten und entsprechenden auf Ihr Risikoprofil abgestützten Anlagestrategien.

Erhalten Sie sich genügend Flexibilität mit dem investierten Kapital, um auf veränderte Markt-, Gesetzes- oder Familiensituationen reagieren zu können. Versicherungen können eine sinnvolle Ergänzung sein und gewähren ein hohes Mass an Sicherheit. Unter bestimmten Voraussetzungen sind diese auch steuerlich privilegiert.

\section{Immobilien}

Die Frage, ob bestehende Hypotheken reduziert oder, wie zum Teil auch empfohlen wird, sogar erhöht werden sollen, sollte gut überlegt und berechnet werden. Vergleichen Sie, was Sie die Hypothek nach Steuern kostet und welchen Ertrag Sie mit dem Kapital nach Steuern erwirtschaften können. Prüfen Sie, welchen Betrag Sie amortisieren können, ohne sich stark einschränken zu müssen. Halten Sie einen Teil Ihrer Hypotheken flexibel, so können Sie auf veränderte Markt- und Zinssituationen reagieren.

\section{Steuern}

Die Renten der AHV wie auch die Renten der Pensionskasse bilden zu 100\% steuerpflichtiges Einkommen. Prüfen Sie, ob anstehende Renovationen an Ihrer Liegenschaft in der Zeit Ihrer Erwerbstätigkeit oder nach der Pensionierung die grössere Steuereinsparung mit sich bringen. Nutzen Sie auch steueroptimierte Anlageprodukte von Banken und Versicherungen. Sie haben zu Ihrer aktiven Zeit die Möglichkeit, fehlende Pensionskassenguthaben oder durch die Frühpensionierung reduzierte Leistungen einzukaufen, prüfen Sie diese Steueroptimierung. Kapitalleistungen aus Vorsorge (Pensionskassen und Säule 3a) werden kantonal unterschiedlich besteuert, prüfen Sie, ob Ihr Wohnkanton verschiedene Auszahlungen zusammen besteuert. Ein gestaffelter Bezug könnte hier einige Steuern sparen.

Wichtig! Stellen Sie nicht die Steuern über alle weiteren Entscheide zu Ihrer Pensionsplanung, sondern optimieren Sie die Steuerbelastung aufgrund Ihrer Planung.

\section{Nachfolge}

Spätestens jetzt ist der Zeitpunkt gekommen, sich mit dem Güter- und Erbrecht auseinanderzusetzen und die notwendigen Schritte zu veranlassen. Alles, was Sie zu Lebzeiten regeln können, hilft, für die Zukunft klare Verhältnisse zu schaffen und für die Hinterbliebenen unangenehme Situationen zu vermeiden. Nutzen Sie die Möglichkeiten, die Ihnen das Erb- und Güterrecht bietet. Regeln Sie in einem Ehevertrag den Güterstand, die Eigengüter und Errungenschaften. Bestimmen Sie in einem Testament, wer was bekommen soll (Liegenschaft, Wertschriften usw.), und setzen Sie gegebenenfalls einen Willensvollstrecker ein. Sollten Sie bereits einen Ehe- und Erbvertrag oder ein Testament aufgesetzt haben, überprüfen Sie, ob die getroffenen Regelungen noch Ihren Wünschen entsprechen. Mit einem Ehe- und Erbvertrag können Sie oft mehr bewirken als mit teuren Versicherungen.

\section{Wann soll die Planung beginnen?}

Es gibt keine verbindliche Altersangabe, jedoch hat die Erfahrung gezeigt, dass, je früher mit der Planung begonnen wird, desto flexibler die Ausgestaltung bleibt und desto mehr Möglichkeiten sich zur Optimierung insbesondere der Steuersituation bieten. Wer weiss schon mit 50 , ob er mit 60 in Frühpensionierung geht oder ob er seine Praxis bis 70 weiterbetreiben wird? Eine flexibel gehaltene und über die Jahre begleitete Planung kann sich den veränderten Rahmenbedingungen und Bedürfnissen anpassen und gibt für die Zukunft Sicherheit. 